\title{
Acknowledgement to Reviewers of European Journal of Investigation in Health, Psychology and Education in 2019
}

\section{EJIHPE Editorial Office}

MDPI, St. Alban-Anlage 66, 4052 Basel, Switzerland

Published: 16 January 2020

The editorial team greatly appreciates the reviewers who have dedicated their considerable time and expertise to the journal's rigorous editorial process over the past 12 months, regardless of whether the papers are finally published or not. In 2019, a total of 26 papers were published in the journal, with a median time to first decision of 17.5 days and a median time from submission to publication of 43 days. The editors would like to express their sincere gratitude to the following reviewers for their generous contribution in 2019:

\begin{tabular}{|c|c|}
\hline Abbas, Azhar & Martin, Stephanie L. \\
\hline Al-Lami, Faris & McCamley, John \\
\hline Allred, Sarah & McClinchy, Jane \\
\hline Andreou, Eleni & McFadden, Sandra \\
\hline Antonopoulou, Vivi & McMillan, Janice \\
\hline Arribas Marín, Juan Manuel & Méndez Mateo, Inmaculada \\
\hline Baez, Yolanda & Mira, José Joaquín \\
\hline Barbosa, David Perez & Mizuno, Koh \\
\hline Barcham, Richard & Mugabi, Ivan K. \\
\hline Bolboacă, Sorana D. & Mukherjee, Soumyadeep \\
\hline Bungum, Timothy & $\mathrm{Na}$, In Seop \\
\hline Burnett, Harvey & Napal, María \\
\hline Burr, Brandon & Natafgi, Nabil \\
\hline C. de la Torre-Montero, Julio & $\mathrm{Ng}$, Qin Xiang \\
\hline Cebrián, Gisela & Nguyen, Khai The \\
\hline Ceccatto, Vânia Marilande & Niakšu, Olegas \\
\hline Chambers IV, Edgar & Nicolaou, Constantinos \\
\hline Choate, Peter W. & Nowacki, Marek \\
\hline Cook, Amanda C. & Núñez-Cacho Utrilla, Pedro \\
\hline Cori, Jennifer & Olushola, Joyce \\
\hline Cupani, Marcos & Ouellette, Rachel R. \\
\hline Dibaba, Daniel & Pecoraro, Fabrizio \\
\hline Didham, Robert & Pérez-Lopez, Alberto \\
\hline Doukakis, Spyros & Podovšovnik, Eva \\
\hline Drigas, Athanasios S. & Potluka, Oto \\
\hline Dziuba, Szymon & Power, Thomas G. \\
\hline
\end{tabular}




\begin{tabular}{|c|c|}
\hline Egea Zerolo, Blanca & Pozo Rico, Teresa \\
\hline Eiroa-Orosa, Francisco José & Raheem, Bamidele \\
\hline El Ghoch, Marwan & Reid, Eva \\
\hline Elmi, Chiara & Reimers, Anne Kerstin \\
\hline Engelhard, Matthew & Reiter, E. Miranda \\
\hline Fang, Di & Rogge, Ronald D. \\
\hline Fernandez Salinero, Samuel & Rudnick, Abraham \\
\hline Field-Richards, Sarah & Ruhle, Sascha \\
\hline Fusheini, Adam & Rutkauskaite, Renata \\
\hline Gardner, Lauren & Ryabov, Igor \\
\hline Gębski, Jerzy & Salessi, Solana \\
\hline Georgalos, Konstantinos & Salmerón-Manzano, Esther \\
\hline Georgiadou, Elissavet Gina & San Pedro Veledo, María Belén \\
\hline Gerke, Oke & Santoro, Paolo Emilio \\
\hline Gómez-de-Terreros-Guardiola, Montserrat & Schindler, Lena \\
\hline Goto, Kenichi & Seeman, Mary V. \\
\hline Guzik, Agnieszka & Serra, Lídia \\
\hline Györkös, Christina & Shah, Ajit \\
\hline Hermanussen, Michel & Siu, Fiona \\
\hline Houdmont, Jonathan & Soares Teles, Ariel \\
\hline Hughes, Vickie & Sperlì, Giancarlo \\
\hline Hurstel, Alexandre & Stará, Jana \\
\hline Irsara, Martina & Steen, S. (Steffie) Van Der \\
\hline Itatani, Tomoya & Ștefan, Simona Cătălina \\
\hline Jones, Lise Øen & Stewart, Thomas \\
\hline Kantono, Kevin & Strickland, Paul \\
\hline Karatsidis, Angelos & Tararova, Olga \\
\hline Katona, Jozsef & Tokarz, Aleksandra \\
\hline Katsanos, Aristeidis H. & Toscano, Ferdinando \\
\hline Kayser, Lars & Toselli, Stefania \\
\hline Kelly, Kimberly & Tyburski, Ernest \\
\hline Kim, Byung-Jik & Ulibarri Ochoa, Ainhoa \\
\hline Kira, Geoff & Van Hoof, Chris \\
\hline Kirchner, Kathrin & Varelas, Vassileios \\
\hline Lauridsen, Jørgen Trankjær & Vila-Vázquez, Guadalupe \\
\hline Lerma, Claudia & Villafaina, Santos \\
\hline Lerman, Dorothea & Volenzo, Tom Elijah \\
\hline Lewko, Jolanta & Voroshilova, Anzhelika \\
\hline Li, Weijuan & Wanchai, Ausanee \\
\hline Liu, Changqi & Weigelt, Oliver \\
\hline Llorente-Alonso, Marta & Wenos, Jeanne \\
\hline Lochbaum, Marc & Wilson, Jason \\
\hline Luis Ubago, Jose & Wren, Douglas \\
\hline
\end{tabular}


M. Silva, Fernanda

Magnavita, Nicola

Marcolin, Federica

Marker, Sandra
Yeh, Jun-Jun

Zakariya, Yusuf F.

Zhao, Shuo

(c) 2020 by the author. Licensee MDPI, Basel, Switzerland. This article is an open access article distributed under the terms and conditions of the Creative Commons Attribution (CC BY) license (http://creativecommons.org/licenses/by/4.0/). 